\title{
Calculation of shallow polymer shell taking the creep into account
}

\author{
Levon Mailyan ${ }^{1}$, Anton Chepurnenko ${ }^{1 *}$, Serdar Yazyev ${ }^{1}$ and Batyr Yazyev ${ }^{1}$ \\ ${ }^{1}$ Don State Technical University, 344022, Sotcialisticheskaya, 162, Rostov-on-Don, Russia
}

\begin{abstract}
In this paper, we obtain the equations allowing the calculation of shallow shells taking the creep into account under an arbitrary law of relationship between creep deformations and stresses. We also consider the methodology of calculation of polymeric membranes, the material of which is subject to a nonlinear equation of Maxwell-Gurevich.
\end{abstract}

\section{Introduction}

Shallow shells are widely used in the technics and particularly in the construction, so their consideration is of great interest in itself. The geometry of the middle surface of shallow shells can be identified with the geometry of the plane on which they are projected. Curvilinear coordinates, along the lines laid by the principal curvatures can be considered to coincide with the Cartesian coordinates in the plane. Problems of calculation of shallow shells in the elastic stage are fairly well understood, but many materials are characterized by not only the elastic, but also viscoelastic properties [1-4]. When creep is taken into account geometrical and static equations of shallow shells theory remain unchanged. Changes occur only in the physical equations.

\section{Derivation of resolving equations}

Let the median surface of the shell, shown at Fig.1 be described by the equation:

$$
z=f(x, y) \text {. }
$$

Full shell deformations are represented as the sum of the middle surface deformations and deformations caused by the change of curvature [5]:

$$
\begin{aligned}
& \varepsilon_{x}=\varepsilon_{x}^{0}+z \chi_{x} ; \\
& \varepsilon_{y}=\varepsilon_{y}^{0}+z \chi_{y} ; \\
& \gamma_{x y}=\gamma^{0}+2 z \chi_{x y} .
\end{aligned}
$$

The deformations of the middle surface are defined as follows [6]:

*Corresponding author: anton_chepurnenk@mail.ru 


$$
\varepsilon_{x}^{0}=\frac{\partial u}{\partial x}+k_{x} w ; \quad \varepsilon_{y}^{0}=\frac{\partial v}{\partial y}+k_{y} w ; \quad \gamma_{0}=\frac{\partial u}{\partial y}+\frac{\partial v}{\partial x}
$$

where $k_{x} \approx-\frac{\partial^{2} z}{\partial x^{2}}, k_{y} \approx-\frac{\partial^{2} z}{\partial y^{2}}-$ principal curvatures.

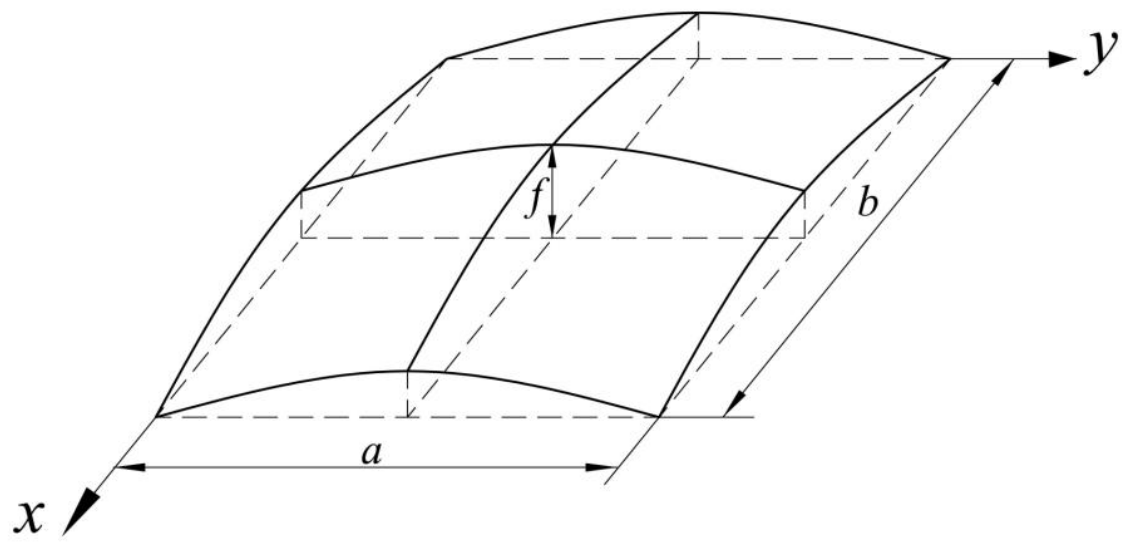

Fig. 1. Shallow rectangular in plan shell

The changes of the curvatures of the middle surface are determined by the same formulas as in the plates:

$$
\chi_{x}=-\frac{\partial^{2} w}{\partial x^{2}} ; \quad \chi_{y}=-\frac{\partial^{2} w}{\partial y^{2}} ; \quad \chi_{x y}=-\frac{\partial^{2} w}{\partial x \partial y} .
$$

Differentiating the first equation in (3) on $x$, and the second - on $y$ and then taking their sum we will get:

$$
\frac{\partial^{2} \varepsilon_{x}^{0}}{\partial y^{2}}+\frac{\partial^{2} \varepsilon_{y}^{0}}{\partial x^{2}}=\frac{\partial^{2} \gamma^{0}}{\partial x \partial y}+\frac{\partial^{2}}{\partial y^{2}}\left(k_{x} w\right)+\frac{\partial^{2}}{\partial x^{2}}\left(k_{y} w\right) .
$$

If $k_{x y}=-\frac{\partial^{2} z}{\partial x \partial y}=0$, that is $k_{x}$ and $k_{y}$ are the principal curvatures, the equation of strain compatibility (5) takes the form:

$$
\frac{\partial^{2} \varepsilon_{x}^{0}}{\partial y^{2}}+\frac{\partial^{2} \varepsilon_{y}^{0}}{\partial x^{2}}=\frac{\partial^{2} \gamma^{0}}{\partial x \partial y}+k_{x} \frac{\partial^{2} w}{\partial y^{2}}+k_{y} \frac{\partial^{2} w}{\partial x^{2}} .
$$

Equilibrium equation in the case when only the vertical load is acting can be written as [5]: 


$$
\begin{aligned}
& \frac{\partial N_{x}}{\partial x}+\frac{\partial S}{\partial y}=0 \\
& \frac{\partial S}{\partial x}+\frac{\partial N_{y}}{\partial y}=0 \\
& \frac{\partial M_{x}}{\partial x}+\frac{\partial H}{\partial y}-Q_{x}=0 \\
& \frac{\partial M_{y}}{\partial y}+\frac{\partial H}{\partial x}-Q_{y}=0 \\
& \frac{\partial Q_{x}}{\partial x}+\frac{\partial Q_{y}}{\partial y}-k_{x} N_{x}-k_{y} N_{y}+q=0
\end{aligned}
$$

where $N_{x}, N_{y}$ - longitudinal forces, $S$ - shear force, $M_{x}, M_{y}$ - bending moments, $H$ torque, $Q_{x}, Q_{y}$ - lateral forces, $q$ - surface load.

We exclude from equations (7) lateral forces:

$$
\frac{\partial^{2} M_{x}}{\partial x^{2}}+2 \frac{\partial^{2} H}{\partial x \partial y}+\frac{\partial^{2} M_{y}}{\partial y^{2}}-k_{x} N_{x}-k_{y} N_{y}+q=0 .
$$

Physical equations are written in the form:

$$
\begin{aligned}
& \varepsilon_{x}=\frac{1}{E}\left(\sigma_{x}-v \sigma_{y}\right)+\varepsilon_{x}^{*} ; \\
& \varepsilon_{y}=\frac{1}{E}\left(\sigma_{y}-v \sigma_{x}\right)+\varepsilon_{y}^{*} ; \\
& \gamma_{x y}=\frac{\tau_{x y}}{G}+\gamma_{x y}^{*}=\frac{2(1+v)}{E} \tau_{x y}+\gamma_{x y}^{*},
\end{aligned}
$$

where $\varepsilon_{x}^{*}, \varepsilon_{y}^{*}, \gamma_{x y}^{*}-$ creep strains.

We express from (9) stresses by the deformations:

$$
\begin{aligned}
& \sigma_{x}=\frac{E}{1-v^{2}}\left(\varepsilon_{x}^{0}+v \varepsilon_{y}^{0}+z\left(\chi_{x}+v \chi_{y}\right)-\left(\varepsilon_{x}^{*}+v \varepsilon_{y}^{*}\right)\right) ; \\
& \sigma_{y}=\frac{E}{1-v^{2}}\left(\varepsilon_{y}^{0}+v \varepsilon_{x}^{0}+z\left(\chi_{y}+v \chi_{x}\right)-\left(\varepsilon_{y}^{*}+v \varepsilon_{x}^{*}\right)\right) ; \\
& \tau_{x y}=\frac{E}{2(1+v)}\left(\gamma^{0}+2 z \chi_{x y}-\gamma_{x y}^{*}\right) .
\end{aligned}
$$

Bending moments and torque are defined as follows: 


$$
\begin{aligned}
& M_{x}=\int_{-h / 2}^{h / 2} \sigma_{x} z d z=D\left(\chi_{x}+v \chi_{y}\right)-M_{x}^{*} ; \\
& M_{y}=\int_{-h / 2}^{h / 2} \sigma_{y} z d z=D\left(\chi_{y}+v \chi_{x}\right)-M_{y}^{*} ; \\
& H=\int_{-h / 2}^{h / 2} \tau_{x y} z d z=D(1-v) \chi_{x y}-H^{*},
\end{aligned}
$$

where $D=\frac{E h^{3}}{12\left(1-v^{2}\right)}-$ cylindrical rigidity,

$$
M_{x}^{*}=\frac{E}{1-v^{2}} \int_{-h / 2}^{h / 2}\left(\varepsilon_{x}^{*}+v \varepsilon_{y}^{*}\right) z d z, \quad M_{y}^{*}=\frac{E}{1-v^{2}} \int_{-h / 2}^{h / 2}\left(\varepsilon_{y}^{*}+v \varepsilon_{x}^{*}\right) z d z, \quad H^{*}=\frac{E}{2(1+v)} \int_{-h / 2}^{h / 2} \gamma_{x y}^{*} z d z .
$$

Longitudinal and shear forces are determined as follows:

$$
\begin{aligned}
& N_{x}=\int_{-h / 2}^{h / 2} \sigma_{x} d z=\frac{E h}{1-v^{2}}\left(\varepsilon_{x}^{0}+v \varepsilon_{y}^{0}\right)-N_{x}^{*} ; \\
& N_{y}=\int_{-h / 2}^{h / 2} \sigma_{y} d z=\frac{E h}{1-v^{2}}\left(\varepsilon_{y}^{0}+v \varepsilon_{x}^{0}\right)-N_{y}^{*} ; \\
& S=\int_{-h / 2}^{h / 2} \tau_{x y} d z=\frac{E h}{2(1+v)} \gamma_{0}-S^{*},
\end{aligned}
$$

where $N_{x}^{*}=\frac{E}{1-v^{2}} \int_{-h / 2}^{h / 2}\left(\varepsilon_{x}^{*}+v \varepsilon_{y}^{*}\right) d z, \quad N_{y}^{*}=\frac{E}{1-v^{2}} \int_{-h / 2}^{h / 2}\left(\varepsilon_{y}^{*}+v \varepsilon_{x}^{*}\right) d z, S^{*}=\frac{E}{2(1+v)} \int_{-h / 2}^{h / 2} \gamma_{x y}^{*} d z$

The first two of the equilibrium equations (7) can be satisfied if we introduce stress function by formulas:

$$
N_{x}=\frac{\partial^{2} \Phi}{\partial y^{2}}, \quad N_{y}=\frac{\partial^{2} \Phi}{\partial x^{2}}, \quad S=-\frac{\partial^{2} \Phi}{\partial x \partial y} .
$$

We express from (12) the middle surface deformations:

$$
\begin{aligned}
& \varepsilon_{x}^{0}=\frac{1}{E h}\left(N_{x}-v N_{y}+N_{x}^{*}-v N_{y}^{*}\right)=\frac{1}{E h}\left(\frac{\partial^{2} \Phi}{\partial y^{2}}-v \frac{\partial^{2} \Phi}{\partial x^{2}}+N_{x}^{*}-v N_{y}^{*}\right) ; \\
& \varepsilon_{y}^{0}=\frac{1}{E h}\left(N_{y}-v N_{x}+N_{y}^{*}-v N_{x}^{*}\right)=\frac{1}{E h}\left(\frac{\partial^{2} \Phi}{\partial x^{2}}-v \frac{\partial^{2} \Phi}{\partial y^{2}}+N_{y}^{*}-v N_{x}^{*}\right) \\
& \gamma^{0}=\frac{2(1+v)}{E h}\left(S+S^{*}\right)=\frac{2(1+v)}{E h}\left(-\frac{\partial^{2} \Phi}{\partial x \partial y}+S^{*}\right) .
\end{aligned}
$$
equation:

Substituting (13) into equation of strain compatibility (6) we obtain the first allowing

$$
\frac{1}{E h} \nabla^{4} \Phi-k_{x} \frac{\partial^{2} w}{\partial y^{2}}-k_{y} \frac{\partial^{2} w}{\partial x^{2}}=\frac{1}{E h}\left(2(1+v) \frac{\partial^{2} S^{*}}{\partial x \partial y}+v\left(\frac{\partial^{2} N_{x}^{*}}{\partial x^{2}}+\frac{\partial^{2} N_{y}^{*}}{\partial y^{2}}\right)-\frac{\partial^{2} N_{x}^{*}}{\partial y^{2}}-\frac{\partial^{2} N_{y}^{*}}{\partial x^{2}}\right)
$$


The second governing equation we obtain by substituting the bending moments (11) in the equilibrium equation (8):

$$
D \nabla^{4} w+k_{x} \frac{\partial^{2} \Phi}{\partial y^{2}}+k_{y} \frac{\partial^{2} \Phi}{\partial x^{2}}=q-\left(\frac{\partial^{2} M_{x}^{*}}{\partial x^{2}}+2 \frac{\partial^{2} H^{*}}{\partial x \partial y}+\frac{\partial^{2} M_{y}^{*}}{\partial y^{2}}\right) .
$$

Thus, the problem of calculation of a shallow shell has reduced to a system of two differential equations of fourth order.

\section{Solution of the problem}

The system of equations (14) and (15) can be solved numerically by the method of finite differences. To determine the creep strain the linear approximation with respect to time can be applied. This approach is used in the papers [7-14]. For polymeric materials we have the nonlinear equation of Maxwell-Gurevich, which for plane stress has the form:

$$
\frac{\partial \varepsilon_{i j}^{*}}{\partial t}=\frac{f_{i j}^{*}}{\eta^{*}}, \quad i=(x, y), j=(x, y),
$$

where $\varepsilon_{i j}^{*}$ - creep deformation, $f_{i j}^{*}-$ stress function, $\eta^{*}-$ relaxation viscosity.

$$
\begin{aligned}
& f_{i j}^{*}=\frac{3}{2}\left(\sigma_{i j}-p \delta_{i j}\right)-E_{\infty} \varepsilon_{i j}^{*} ; \\
& \frac{1}{\eta^{*}}=\frac{1}{\eta_{0}^{*}} \exp \left(\frac{\left|f_{\max }^{*}\right|}{m^{*}}\right),
\end{aligned}
$$

where $p=\left(\sigma_{x}+\sigma_{y}\right) / 3-$ mean stress, $\delta_{i j}-$ Kronecker symbol, $E_{\infty}-$ high elasticity modulus, $m^{*}$ - speed modulus, $\eta_{0}^{*}-$ the initial relaxation viscosity.

It is necessary to note, that $\varepsilon_{x y}^{*}=\frac{1}{2} \gamma_{x y}^{*}$.

If the material of shell is subject to the equation of Maxwell-Gurevich, it is possible to determine the deflection of the shell at the end of the creep process, without resorting to numerical methods. To do this, we analyze the Maxwell-Gurevich equation for $t \rightarrow \infty$. If the creep is damped, at the end of the process the growth rate of all components of creep deformation must be equal to zero:

$$
\begin{aligned}
& \frac{\partial \varepsilon_{x}^{*}}{\partial t}=0 \rightarrow f_{x}^{*}=\frac{3}{2}\left(\sigma_{x}-\frac{\sigma_{x}+\sigma_{y}}{3}\right)-E_{\infty} \varepsilon_{x}^{*}=\sigma_{x}-\frac{\sigma_{y}}{2}-E_{\infty} \varepsilon_{x}^{*}=0 ; \\
& \frac{\partial \varepsilon_{y}^{*}}{\partial t}=0 \rightarrow f_{y}^{*}=\frac{3}{2}\left(\sigma_{y}-\frac{\sigma_{x}+\sigma_{y}}{3}\right)-E_{\infty} \varepsilon_{y}^{*}=\sigma_{y}-\frac{\sigma_{x}}{2}-E_{\infty} \varepsilon_{y}^{*}=0 ; \\
& \frac{\partial \varepsilon_{x y}^{*}}{\partial t}=0 \rightarrow f_{x y}^{*}=\frac{3}{2} \tau_{x y}-E_{\infty} \varepsilon_{x y}^{*}=0 .
\end{aligned}
$$

From the relations (17) we can find maximal creep strains: 


$$
\begin{aligned}
& \varepsilon_{x, \infty}^{*}=\frac{1}{E_{\infty}}\left(\sigma_{x}-\frac{\sigma_{y}}{2}\right) ; \\
& \varepsilon_{y, \infty}^{*}=\frac{1}{E_{\infty}}\left(\sigma_{y}-\frac{\sigma_{x}}{2}\right) ; \\
& \gamma_{x y, \infty}^{*}=\frac{3 \tau_{x y}}{E_{\infty}}=\frac{\tau_{x y}}{G_{\infty}} .
\end{aligned}
$$

The relationship between the total strains and stresses at $t \rightarrow \infty$ takes the form:

$$
\begin{aligned}
& \varepsilon_{x}=\sigma_{x}\left(\frac{1}{E_{\infty}}+\frac{1}{E}\right)-\sigma_{y}\left(\frac{1}{2 E_{\infty}}+\frac{v}{E}\right)=\alpha \sigma_{x}-\beta \sigma_{y} ; \\
& \varepsilon_{y}=\sigma_{y}\left(\frac{1}{E_{\infty}}+\frac{1}{E}\right)-\sigma_{x}\left(\frac{1}{2 E_{\infty}}+\frac{\nu}{E}\right)=\alpha \sigma_{x}-\beta \sigma_{y} ; \\
& \gamma=\tau_{x y}\left(\frac{1}{G_{\infty}}+\frac{1}{G}\right) .
\end{aligned}
$$

These relations can be rewritten as:

$$
\begin{aligned}
& \varepsilon_{x}=\frac{1}{\tilde{E}}\left(\sigma_{x}-\tilde{v} \sigma_{y}\right) ; \\
& \varepsilon_{y}=\frac{1}{\tilde{E}}\left(\sigma_{y}-\tilde{v} \sigma_{x}\right) ; \\
& \gamma=\frac{2(1+\tilde{v}) \tau_{x y}}{E},
\end{aligned}
$$

where $\tilde{E}=\frac{1}{\alpha}-$ long modulus, $\tilde{v}=\frac{\beta}{\alpha}-$ long-term Poisson's ratio.

The system of resolving equations at $t \rightarrow \infty$ takes the form:

$$
\begin{aligned}
& \frac{1}{\tilde{E} h} \nabla^{2} \nabla^{2} \Phi-k_{x} \frac{\partial^{2} w}{\partial y^{2}}-k_{y} \frac{\partial^{2} w}{\partial x^{2}}=0 ; \\
& D_{\infty} \nabla^{2} \nabla^{2} w+k_{x} \frac{\partial^{2} \Phi}{\partial y^{2}}+k_{y} \frac{\partial^{2} \Phi}{\partial x^{2}}=q,
\end{aligned}
$$

where $D_{\infty}=\frac{\tilde{E} h^{3}}{12\left(1-\tilde{v}^{2}\right)}=\frac{\alpha h^{3}}{12\left(\alpha^{2}-\beta^{2}\right)}-$ long cylindrical rigidity, which was first introduced in [14].

Next we will give an example of the calculation of shallow rectangular in plane shell, the middle surface of which is elliptic paraboloid (Figure 1). The equation of this surface can be written as:

$$
z=f\left[\frac{f_{1}}{f}\left(2 \frac{x}{a}-1\right)^{2}+\frac{f_{2}}{f}\left(2 \frac{y}{b}-1\right)^{2}-1\right],
$$

where $f=f_{1}+f_{2}$.

In the calculation it was assumed that at the edges the shell is connected to the diaphragms completely rigid in their plane and flexible out of it. The calculation was performed under the following initial data: $h=0.01 \mathrm{~m}, a=b=1 \mathrm{~m}, f_{1}=f_{2}=0.05 \mathrm{~m}$, the shell material - secondary PVC, $E=1480 \mathrm{MPa}, E_{\infty}=5990 \mathrm{MPa}, \mathrm{m}^{*}=12.6 \mathrm{MPa}, \eta_{0}^{*}=$ $9.06 \cdot 10^{5} \mathrm{MPa} \cdot \min , v=0.3[15]$. The shell was loaded by a permanent, uniformly distributed 
over the area load $q=0.5 \mathrm{kPa}$. Fig. 2 is a plot of distribution of stresses $\sigma_{\mathrm{x}}$ depending on $x$ and $y$ at $z=-h / 2$. Net surface corresponds to the time $t=0$, shaded $-t=10 \mathrm{~h}$.

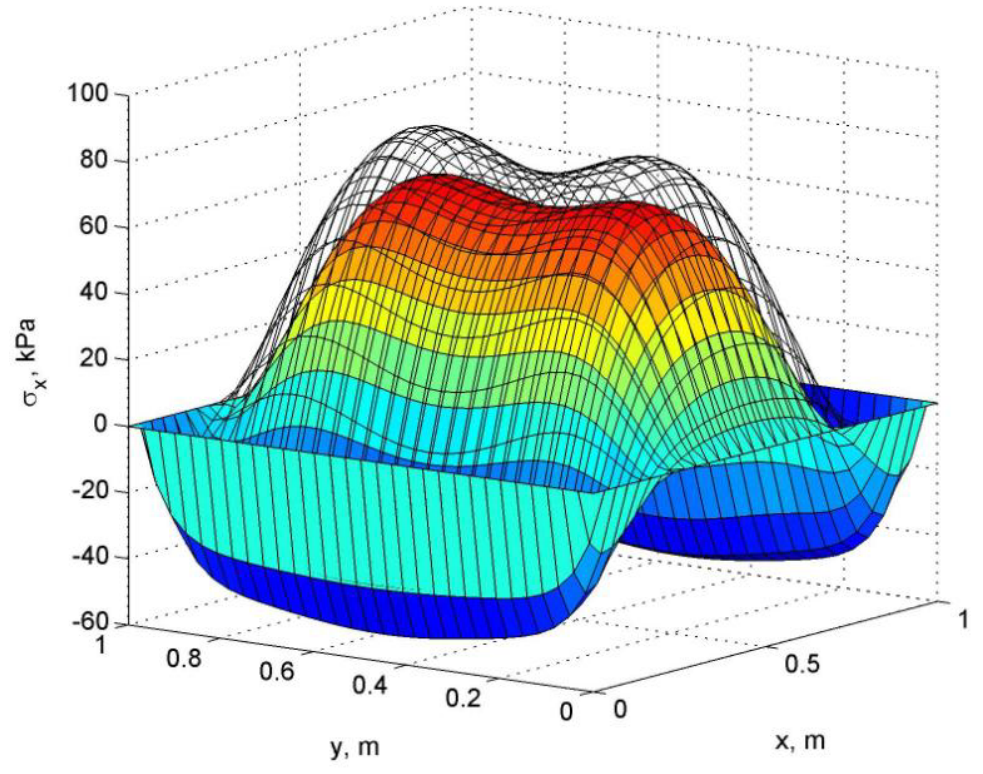

Fig. 2. The stress distribution at $z=-h / 2$ : net surface $-t=0$, shaded surface $-t=10 \mathrm{~h}$.

Fig. $3-\sigma_{\mathrm{x}}$ stress distribution as a function of $x$ and $y$ at $z=h / 2$. Net surface is the result at $t=10 \mathrm{~h}$, shaded - at $t=0$.

From Fig. 2 and 3 we can see that the stress in the bottom surface of the shell increases and in the upper surface stress decreases. The maximum stress at the beginning and end of creep process differ by $19 \%$.

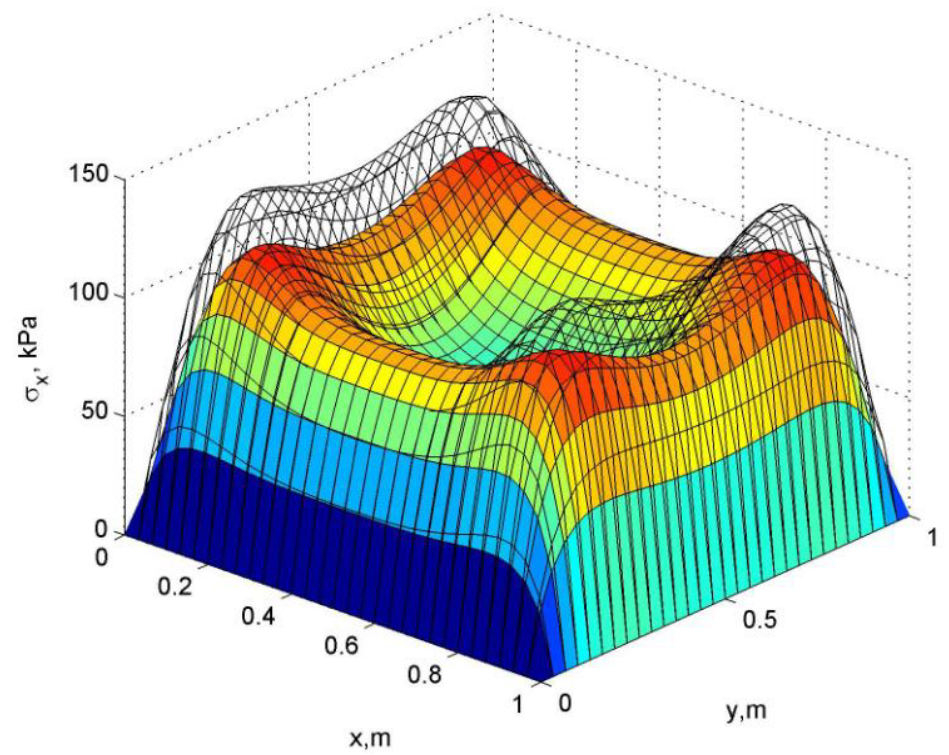

Fig. 3. The stress distribution at $z=h / 2$ : net surface $-t=10 \mathrm{~h}$, shaded surface $-t=0$. 


\section{Summary}

Obtained equations allow calculation of shallow shells of any form on any load at an arbitrary law of the connection between stresses and creep strain. For polymers, obeying the Maxwell-Gurevich law it is possible to calculate deflections and stresses in the end of the creep process, having only the solution of the elastic problem.

\section{References}

1. J. Betten. Creep mechanics (Springer Science \& Business Media, 2008)

2. M.F. Ashby, B.F. Dyson. Creep damage mechanics and micromechanisms (ICF6, New Delhi, 1984 (2013)

3. J. T. Boyle, J. Spence. Stress analysis for creep (Elsevier, 2013)

4. A. K. Miller. Unified constitutive equations for creep and plasticity (Springer Science \& Business Media, 2012)

5. A.L. Gol'Denveizer. Theory of Elastic Thin Shells: Solid and Structural Mechanics. (Elsevier, 2014)

6. H. Altenbach, V.A. Eremeev, N. F. Morozov. Mechanics of Solids, 45, 331-342 (2010)

7. B. F. Oliveira, G. J. Creus. Composite Structures, 65, 443-457 (2004)

8. B. Miled, I. Doghri, L. Delannay. Computer Methods in Applied Mechanics and Engineering, 200 3381-3394 (2011)

9. R. M. Haj-Ali, A. H. Muliana, International Journal for Numerical Methods in Engineering, 59, 25-45 (2004)

10. J. G. J. Beijer, J. L. Computers \& structures, 80, 1213-1229 (2002)

11. J. S. Kim, A. H. Muliana. International journal for numerical methods in engineering, 79, 550-575 (2009)

12. V.I. Andreev, A.S. Chepurnenko, B.M. Yazyev. Advanced Materials Research, 10041005, 257-260 (2014)

13. V.I. Andreev, B.M. Yazyev, A.S. Chepurnenko, Advanced Materials Research, 900 707-710 (2014)

14. A.S. Chepurnenko, B.M. Yazyev, A.A. Savchenko, Procedia Engineering, 150, 16801685 (2016)

15. A.S. Chepurnenko, V.I. Andreev, A.N. Beskopylny, B.M. Jazyev, MATEC 67, 06059 (2016) 